\title{
Enhanced Solubility of Modafinil via Solubilization Techniques
}

\author{
Juturu Thimmasetty', Tanmoy Ghosh ${ }^{2 *}$, Shashank Nayak Nagar', Shwetha Kamath', Shanmuganathan Seetharaman², Abbas Kumai \\ Mohamed ${ }^{1}$ \\ 'Department of Pharmaceutics, Bapuji Pharmacy College, Davangere, Karnataka, INDIA. \\ 2Department of Pharmaceutics, Faculty of Pharmacy, MS Ramaiah University of Applied Sciences, Bangalore, Karnataka, INDIA. \\ ${ }^{3}$ Department of Pharmaceutics, Faculty of Pharmacy, Sri Ramachandra Institute of Higher Education and Research (Deemed to be University), Porur, Chennai, Tamil \\ Nadu, INDIA.
}

\begin{abstract}
Objectives: The objective of the study was to compare solubilization approaches, namely cosolvency, micelle solubilization, complexation, pH adjustment and hydrotrophy, on BCS class II drug modafinil solubility. Methods: Altered solubility study of modafinil was carried out in numerous water-cosolvent mixtures. Similar type of study was performed using different ionic and non-ionic surfactants using phase solubility analysis. Furthermore, solubility behavior with two cyclodextrins namely beta cyclodextrin and maltodextrin were examined. Solubility studies were conducted in buffers of different $\mathrm{pH}$ ranging from 1.2 to 8 at different temperatures $\left(25^{\circ} \mathrm{C}\right.$ and $\left.37^{\circ} \mathrm{C}\right)$. The effect of hydrotrophy on the solubility of modafinil was also studied. Results: Outcomes showed that ethanol is depicted to enhance solubility by greater height. It is found that ionic surfactants were better solubilizers than non-ionic surfactants. The capacity of solubilization is depicted to enhance with rise in hydrocarbon chain length of surfactant, denoting that hydrocarbon core of micelles as the center of solubilization. Highest solubility and binding constants were achieved with
\end{abstract}

use of beta cyclodextrins followed by maltodextrins. The buffers of $\mathrm{pH} 2$ and $\mathrm{pH} 3$ have shown highest solubility when compared to others. Sodium thiocyanate salt had shown highest solubility of the drug. Conclusion: As a consequence, the study provides dataset so as to contrast effects of numerous solubilizers on modafinil solubility and also gives an insight of the mode of solubilization by aforementioned techniques.

Key words: Modafinil, Complexation, Cosolvency, Hydrotrophy, Solubility, Surfactants.

\section{Correspondence}

\section{Mr. Tanmoy Ghosh}

Department of Pharmaceutics, Faculty of Pharmacy, MS Ramaiah University of Applied Sciences, Bangalore-560054, Karnataka, INDIA.

Phone: +918867763868

Email: tanmoy.ps.ph@msruas.ac.in

DOI: 10.5530/jyp.2020.12.26

\section{INTRODUCTION}

The drugs to be made soluble in numerous solvents along with water are pivotal for the drug to be developed and synthesized. The information has it's crucial role to evaluate the pharmacokinetic and toxicological nature of drugs. The poor aqueous solubility and wettability of drug showcases the probable challenges in the design of myriad pharmaceutical dosage form and lead to altered oral bioavailability. Solubilization is defined as the preparation of a stable thermodynamic solution of a substance that is basically very slightly soluble or may be insoluble in a given solvent, by encapsulating numerous amphiphilic component(s). ${ }^{1}$ Buffers, cosolvency, surfactants, $\mathrm{pH}$ adjustment, complexation and hydrotrophy are frequently used pharmaceutical techniques for solubilizing drugs with depleted aqueous solubility. ${ }^{2}$

Utilization of cosolvent is one of the frequently used techniques for the processes of separation, isolation and crystallization of the given active pharmaceutical ingredient. Trial and error approach is most widely used for selecting the volume of solvent required to dissolve the drug. It will be extremely useful if an appropriate mixing ratio of the solvent system that dissolves the drug is known. The data on the solubilities of the drug in the mixed solvent system with varied composition is scanty. Although it is highly competent, its use might be close by limit of concentration of cosolvent that can be utilized and its ability of precipitation with the dilution. The researchers have thrown light on utilization of cosolvents along with surfactants and cyclodextrins. ${ }^{3,4}$

Micellar approach of solubilization utilizing surfactant is one of the historic and vigorous techniques to improve solubilization. With arrival of nonionic surfactant, with nadir CMC, affinity with biological system and elevated solubilizing capacity, has been sky rocketing of late. These surfactants, in the aqueous habitat, engulf together to create micelles which can be stated as two region system, internal nonpolar region of hydrocabon part and external capsular part of polyoxyethylene chains. The internal hydrophobic core and exterior interfacial region known as mantle is usually the fixed point of solubilization of the drugs, which are non-polar. ${ }^{5}$

Cyclodextrins are playing an indispensable role as solubilizer and these are obtained naturally from hydrophilic derivative. Cyclodextrins (CDs) have the capability to form inclusion complex with small molecule in host. Furthermore, it inturn affects many properties of drug moiety; most crucial among them is the elevation of solubility., 4 The most commonly used complexing agents in solubilization technology are $\beta$-cyclodextrin, maltodextrin, hydroxypropyl- $\beta$-cyclodextrin, and methyl- $\beta$-cyclodextrin. ${ }^{7}$

$\mathrm{pH}$ modification is a simplest and tailored procedure to improve solubility. One among the vital factors which is known for dissolution of organic compound is its capacity to breakdown into ionic moiety, this is interdependent on the $\mathrm{pH}$ of the utilized medium. The development of liquid and parenteral dosage form has direct relationship with the solubility based on $\mathrm{pH}^{8}$

Since numerous approaches with regard to drug development or application take place in companionship of differing concentrations of different types of salts, the action of salts on solubility of drug is 
indispensable. Analyzing the solubilities of a drug by incorporation of various salts is challenging. Hence, determination of the solubility of drug in presence of different salts is of a great help. Effect of salts (hydrotrophy) on solubility of caffeine in water has been reported. ${ }^{9}$

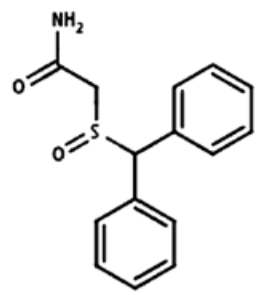

Modafinil is chemically designated as 2-benzhydrl sulfinyl ethanamide, indicated for treatment of narcolepsy. It is desirable to improve modafinil solubility, since modafinil is a poorly aqueous soluble drug ${ }^{10}$ by using various solubilization techniques to improve its pharmacokinetic aspects. Though modafinil is currently available as solid dosage form, its use as liquid dosage forms cannot be ruled out either as oral products or as injections. It is known fact that liquid orals undergo absorption at a faster rate than solid dosage form, as dissolution is the controlling and additional step in the latter. Similar is the case with parenteral. Despite in depth research work carried out utilizing different methods of solubilization, still a comparative profile is very less. The aim of present work is to analyze effect of various solubilization approaches utilizing cosolvency, solubilization by micellar approach, cyclodextrin complexation, $\mathrm{pH}$ adjustment, hydrotrophy, on aqueous solubility of the drug modafinil and comparison thereafter. An approach has been attempted to look into mode of solubilization by above mentioned techniques.

\section{MATERIALS AND METHODS}

Modafinil was procured as a gift sample from Matrix Laboratories (Hyderabad, India). Brij 35, Tween 80 and SLS (sodium lauryl sulphate) were purchased from Merck Chemicals (Mumbai, India). All other chemicals and solvents used were of analytical grade. Water used was double distilled in all glass apparatus in the lab. The remaining solvents purchased from the market were of analytical grade and were used as such.

The maximum absorption $\left(\lambda_{\text {max }}\right)$ of modafinil in $0.1 \mathrm{~N}$ sodium hydroxide solution was found at $218 \mathrm{~nm}$. The concentration utilized was in the concentration range of $2-10 \mu \mathrm{g} / \mathrm{ml}$, which obeyed Beer-Lambert law and the calibration curve was constructed $\left(R^{2}=0.9971\right)$.

\section{Solubility studies}

The method adopted by Higuchi and Connors was used to perform solubility study. ${ }^{11}$ A slightly higher proportion of modafinil was added to $25 \mathrm{ml}$ flasks having $10 \mathrm{ml}$ of various blends of water-cosolvent (ethanol, PG, EG, PEG 400, PEG 600 and glycerin) mixtures (0-100 $\% \mathrm{v} / \mathrm{v})$ to study the effect of cosolvent. Among the cosolvents used, ethanol, propylene glycol, glycerin and polyethylene glycol are used in injectable preparations. ${ }^{12}$ Flasks were later shaken in cryostat at constant temperature using shaker bath for one day at $25^{\circ}$ in order to achieve equilibrium. Preliminary experiments involving repetitive sampling and analysis were performed to ensure equilibrium of the saturated solutions. After achieving equilibrium, aliquots were then withdrawn and filtered utilizing $0.22 \mu \mathrm{m}$ pore size. The concentration of the drug modafinil in the saturated solution was then analyzed after appropriate dilution using suitable solvent by ultraviolet absorption spectroscopy at $218 \mathrm{~nm}$ using UV/Visible spectrophotometer (UV-1700 PC, Shimadzu, Japan). The solubility experiments were carried out in triplicate.
Further, for solubility studies, instead of cosolvent mixtures; increasing concentrations in the range of 0.3 to $1.5 \% \mathrm{w} / \mathrm{v}$ of surfactant (cetrimide, SLS, Tween 80 , Brij 35 and poloxamer F 68 ) solutions were taken to study the effect of micellization. To investigate the effect of complexation on modafinil solubility, the solutions of $\beta$-cyclodextrin in the concentrations range of 0.3 to $1.5 \% \mathrm{w} / \mathrm{v}$ in water were used. Similar concentrations were used even for maltodextrins. To study the effect of $\mathrm{pH}$ on modafinil solubility, the solutions of buffers of $\mathrm{pH}$ ranging from 1.2 to 8 were used. To interpret the effect of temperature on modafinil solubility in various buffers, the solubility studies were conducted at $25^{\circ}$ and $37^{\circ} \mathrm{C}$. Finally, the effect of hydrotrophy was studied using salts such as sodium chloride, sodium bromide, sodium thiocyanate and sodium sulphate of different concentrations $(0-1 \% \mathrm{w} / \mathrm{v})$.

\section{RESULTS}

A solid drug will not be absorbed to appreciable extent across the GI barriers unless it is in solution. Moreover, many pharmaceutical formulations require the addition of a solubilizer to enhance the solubility of sparingly soluble components. The solubility of modafinil was studied in water alone and in combination with several solubilizing agents. Non-polar nature of modafinil does not break and therefore cannot accommodate the entire molecule in the free lattice space of water resulting in less water solubility. However high value of modafinil partition coefficient in $n$-octanol-water (experimentally determined log $P=5.3165)$ suggests good solubility in lipophilic solvents.

\section{Cosolvency}

Cosolvent addition is a highly effective technique for enhancement of solubility of poorly soluble drugs. The small non-polar hydrocarbon region in the cosolvent can reduce the ability of the aqueous system to squeeze out nonpolar solutes. The solubility of modafinil is studied in different cosolvents with a view to enhance the solubility in water. Widely used water miscible cosolvents in the pharmaceutical industry, ethanol, PG, EG, glycerin, PEG 400 and PEG 600, were utilized during the study for the purpose of enhancement of modafinil solubility. The solvent raising more drug solubility is indicated as the stronger solvent and on the other hand the solvent with low solubilization power is regarded as the weaker solvent. Dielectric constants of the solvents reported in the literature are given in Table 1 . Solubility trends of modafinil in watercosolvent systems are shown in Figure 1 (EG, PEG 400 and PEG 600) and Figure 2 (ethanol, PG and glycerin). The $\sigma$ values for the various water-cosolvent systems are given afterwards in Table 2 .

$\varepsilon_{\text {mix }}=\varepsilon_{\text {ws }} f_{\text {ws }}+\varepsilon_{\text {ss }} f_{\text {ss }}$

Where $\varepsilon$ represents dielectric constant, $f$ for volume fraction, mix for mixture, ws for weaker solvent and ss for stronger solvent. The effect of decrease in the dielectric constant of water is directly proportional to the quantity of cosolvent present in the blend, which ultimately increased the drug's solubility most of the times. The relation between the increased solubility of the drug and cosolvent proportion in the solvent blend can be calculated with the equation typed below ${ }^{11}$

$\log \left[D_{\text {tot }}\right]=\log \left[D_{u}\right]+\sigma[C]$

Where, $\left[D_{\text {tot }}\right]$ and $\left[D_{u}\right]$ represent the total observed drug solubility and the intrinsic drug solubility, respectively, $[C]$ and $\sigma$ represent the cosolvent proportion and the solubilization power of the cosolvent, respectively.

\section{Micellar solubilization}

Surfactants are being used to enhance the solubility of drugs as the former form the aggregates called micelles which encapsulate the latter inside them. The surfactants used for micellar solubilization of the drugs 
are cationic, anionic and nonionic. ${ }^{13}$ Non-ionic surfactants are more biocompatible than ionic surfactants. Solubility trends of modafinil in presence of different surfactants are shown in Figure 3. Using the slope of the phase solubility diagram, molar solubilization capacity was calculated (solubility of the drug in $\mathrm{mg} /$ surfactant in $\mathrm{g}$ ). $S_{\text {free }}$ was calculated from the relation; $S_{\text {free }}=$ Intercept + (slope x CMC estimated from the graph). The distribution coefficient $\left(K_{m}\right)$ was calculated thereafter using the relation, $K_{m}=$ Slope $/ S_{\text {free }}{ }^{11,14}$

Distribution coefficients of various surfactants are given in Table 3.

Amphiphilic nature of surfactants is due to the presence of hydrophilic and lipophilic portions in their structures. All surfactants resulted in enhancement of aqueous solubility of modafinil. As anticipated the solubility of modafinil increased with increase in surfactant concentration. This implies that solubility follows partition model. ${ }^{15}$ The order of solubility enhancement is as follows, cetrimide (324 fold) $>$ SLS (68 fold) $>$ Tween 80 (52 fold) > Brij 35 (6 fold) > poloxamer F 68 (4 fold). Modafinil is positively charged molecule. Cetrimide being cationic surfactant rejects the positively charged modafinil. This facilitates the modafinil to move into micelle. As SLS is anionic surfactant, some of the drug gets adsorbed on micelle surface by electrostatic attraction between negatively charged SLS and positively charged modafinil. Tween 80 is nonionic surfactant. The bulk of modafinil is more. Hence modafinil is not present between poly oxy ethylene chains. Even on the surface also the drug is not adsorbed as there is no charge. The modafinil to a lesser extent goes into the micelle. Ionic surfactants such as cetrimide and sodium lauryl sulphate exhibited greater modafinil solubility than the non-ionic surfactants Tween 80, Brij 35 and poloxamer F 68. Ionic surfactants proved them better than the non-ionic due to presence of ionic interactions between drug and ionic surfactants along with micellar solubilization effect.

Perusal to the Table 2 indicates that distribution coefficient increases with the increase in the solubilization efficiency. The results reflect that higher concentration of drug is entrapped by the micellar core of surfactants. As a result the molecules of the drug are solubilized by micellar solubilization. If the drug is non polar in nature, then it tends to be entrapped in the centre of micelle or near the core.

The total solubility of the drug can be calculated using the below typed equation;

$S_{\text {total }}=S_{w}+k\left(C_{\text {surf }}-\mathrm{CMC}\right)$

Where, $C_{\text {surf }}$ represents the surfactant concentration, CMC represents critical micelle content and $k$ represents the molar solubilization capacity. If the $\mathrm{CMC}$ is much lower than $C_{\text {surf }}$, equation (3) can be modified as follows;

$S_{\text {total }}=S_{w}+k C_{\text {surf. }}$

Solubilization capacities calculated using this equation are given in Table 3. Highest micellar solubilization capacity was shown by cetrimide. Tween 80 , possesses the HLB value of 15 and longer hydrocarbon chain length than Brij 35, exhibited greater solubilization power. On the otherhand, Poloxamer exhibited least effect which could be attributed to its higher HLB value and short hydrophobic chain.

\section{Complexation}

The complexation of modafinil with $\beta$-cyclodextrin and maltodextrin is investigated by phase solubility studies. Figure 4 reflects the solubility profile of modafinil with $\beta$-cyclodextrin and maltodextrin.

\section{$\mathrm{pH}$ modification}

The solubility of modafinil in different buffers of $\mathrm{pH}$ values ranging from 1.2 to 8.0 was determined at $25^{\circ}$ and $37^{\circ} \mathrm{C}$. Absorption of modafinil is influenced by its surroundings in the GI tract. The aqueous solubility of modafinil in the buffers was influenced by the $\mathrm{pH}$ of the buffer. The solubility profiles of modafinil in buffers at $25^{\circ}$ and $37^{\circ}$ are shown in Figure 5. The ratios of drug solubility in water to buffers are shown in Table 3. Due to very less solubility of modafinil in water, exact figures are often difficult to determine, which is in accordance with similar drugs including meloxicam as evidenced in the literature. ${ }^{16,17}$

To explore the effect of temperature on the modafinil solubility in buffer, the solubility study was performed at two temperature levels i.e. $25^{\circ}$ and $37^{\circ}$. Elevation of the temperature resulted in the increase in the solubility of modafinil (Figure 5). The solubility of modafinil as in case of solids in general associated with temperature (endothermic) due to the lowered stability of the crystal lattice.

The structure of modafinil contains ionizable group such as $\mathrm{NH}_{2}$ group. The modafinil is weakly basic in nature and therefore not affected in alkaline medium but has undergone maximum dissociation in acidic $\mathrm{pH}$ of 3. It indicates that the drug has a $\mathrm{pKa}$ value around 3. Further this value is supported by the $\mathrm{pKa}$ value of aureomycin as the latter also has $\mathrm{NH}_{2}$ group and possesses $\mathrm{pKa}$ of $3.3 .{ }^{18}$

\section{Hydrotrophy}

As many steps related to the manufacture of dosage forms are carried out in the presence of different proportions of various salts, effect of salt on solubility of drug is principally important. Determination of solubility of the modafinil in presence of different salts is also a challenging task. Recently the salt effects on solubilities of drugs were predicted. ${ }^{19}$ The effects of $\mathrm{NaCl}, \mathrm{NaBr}, \mathrm{NaSCN}$ and $\mathrm{Na}_{2} \mathrm{SO}_{4}$ were studied and the solubility profiles are shown in Figure 6.

\section{DISCUSSION}

The use of cosolvent is an alternative technique for achieving increased solubility of drugs with insufficient solubility. ${ }^{14,20,21}$ Reduction of the squeezing nature of the water by the addition of the cosolvent is attributed to the non-polar part of the cosolvent. More drug molecules of non-polar nature can go into the solution of less polarity that is created due to the cosolvent which decreases the chemical potential of the solution as the density of the hydrogen bonds also decreases. With the decrease in the polarity of the solvent, solubilization power increases as a result $\sigma$ values also increase. This is proved true with PEG, the less polar solvent. Exponential increase in the solubility was noticed with increase in the proportion of the cosolvents, PEG 400 and PEG 600. In common, solubility of the drug is directly proportional to the fraction of the cosolvent in the solvent blend with much higher solubilization power at higher cosolvent fraction in the solvent blend. This effect resulted in a positive deviation in the solubility phase diagrams as shown in Figure 1 and Figure 2. This finding is in agreement with the solubility trends of indomethacin..$^{22,23}$ It indicates that modafinil gets solubilized in nonpolar solvent rather than polar solvent.

Commonly, alcohols are preferred over water as solvents. Increase in solubility is observed starting ethylene glycol to propylene glycol. The higher solubility of modafinil in ethanol than in ethylene glycol reveals that the solubility is also influenced by the intermolecular interactions between the solvent molecules. These interactions are usually more in glycols compared to alcohols. Further the increase in modafinil solubility right from ethylene glycol to propylene glycol reveals that the hydrophobic interactions are vital in governing the solubility of the modafinil in glycols. Perusal to Figure 1 indicates that high solubility of drug in water-PEG-600 mixtures compared to water-PEG-400 mixtures indicates that extensive hydrophobic interactions with the PEG 600 compared to PEG 400 as the former has a longer nonpolar part compared with the latter. 
Table 1: Dielectric constants of solvents and solubilities of modafinil in various vehicles at $25^{\circ} \mathrm{C}$.

\begin{tabular}{ccc}
\hline Solvent & Dielectric constant, $\varepsilon$ & Solubility $(\mathrm{mg} / \mathrm{ml})$ \\
\hline Water & 78.36 & 0.0140 \\
Ethanol & 24.30 & 2044.9791 \\
PG & 32.00 & 953.4519 \\
EG & 37.7 & 29.6374 \\
PEG 400 & 12.40 & 75.2266 \\
PEG 600 & 12.40 & 56.5028 \\
Glycerin & 42.50 & 71.2169 \\
\hline
\end{tabular}

Table 2: Distribution coefficient of modafinil in surfactant solutions.

\begin{tabular}{cc}
\hline Surfactant & Distribution coefficient $\left(\boldsymbol{K}_{\boldsymbol{m}}\right)$ \\
\hline Brij 35 & 4.5882 \\
Tween 80 & 3.3488 \\
SLS & 3.6958 \\
Poloxamer & 2.4071 \\
Cetrimide & 8.5335 \\
\hline
\end{tabular}

$\sigma=$ cosolvent solubilizing power; $k=$ molar solubilization capacity; $K=$ stability constant; $b=$ ratio of drug solubility in water to buffer; $k_{s}=$ salting coefficient.

Table 3: Solubilization capacities of different solubilizers for modafinil.

\begin{tabular}{|c|c|c|}
\hline Solubilizer & Concentration range & Parameter \\
\hline PEG 400 & $0-100 \% \mathrm{v} / \mathrm{v}$ & $\sigma=6.9234$ \\
\hline PEG 600 & $0-100 \% \mathrm{v} / \mathrm{v}$ & $\sigma=7.3458$ \\
\hline PG & $0-100 \% \mathrm{v} / \mathrm{v}$ & $\sigma=8.1760$ \\
\hline $\mathrm{EG}$ & $0-100 \% \mathrm{v} / \mathrm{v}$ & $\sigma=4.2453$ \\
\hline Glycerin & $0-100 \% \mathrm{v} / \mathrm{v}$ & $\sigma=4.5610$ \\
\hline Ethanol & $0-100 \% \mathrm{v} / \mathrm{v}$ & $\sigma=5.0414$ \\
\hline Brij 35 & $0.3-1.5 \mathrm{w} / \mathrm{v}$ & $k=0.0578$ \\
\hline Tween 80 & $0.3-1.5 \mathrm{w} / \mathrm{v}$ & $k=0.4786$ \\
\hline SLS & $0.3-1.5 \mathrm{w} / \mathrm{v}$ & $k=0.6884$ \\
\hline Poloxamer F 68 & $0.3-1.5 \mathrm{w} / \mathrm{v}$ & $k=0.0435$ \\
\hline Cetrimide & $0.3-1.5 \mathrm{w} / \mathrm{v}$ & $k=3.5798$ \\
\hline$\beta$ cyclodextrin & $0.3-1.5 \mathrm{w} / \mathrm{v}$ & $K_{c}=15.7920$ \\
\hline Maltodextrin & $0.3-1.5 \mathrm{w} / \mathrm{v}$ & $K_{c}=1.7500$ \\
\hline Buffer & $\mathrm{pH}, 2\left(25^{\circ}\right)$ & $b=21.30$ \\
\hline Buffer & $\mathrm{pH}, 3\left(25^{\circ}\right)$ & $b=25.24$ \\
\hline $\mathrm{NaCl}$ & $0.0-1.0 \mathrm{w} / \mathrm{v}$ & $k_{s}=0.8489$ \\
\hline $\mathrm{NaBr}$ & $0.0-1.0 \mathrm{w} / \mathrm{v}$ & $k_{s}=1.8666$ \\
\hline $\mathrm{NaSCN}$ & $0.0-1.0 \mathrm{w} / \mathrm{v}$ & $k_{s}=5.4741$ \\
\hline $\mathrm{Na}_{2} \mathrm{SO}_{4}$ & $0.0-1.0 \mathrm{w} / \mathrm{v}$ & $k_{s}=0.7169$ \\
\hline
\end{tabular}

Summarizing the results of surfactants reveals that micellar solubilization is directly related to the length of hydrocarbon chain of non-ionic surfactants. From this, it can be interpreted that the modafinil molecules preferably gets solubilized in hydrophobic portion of micelles. The locus of solubilization can be anticipated to be inner hydrophobic core of micelles. Similar results were seen in case of diazepam and prazepam

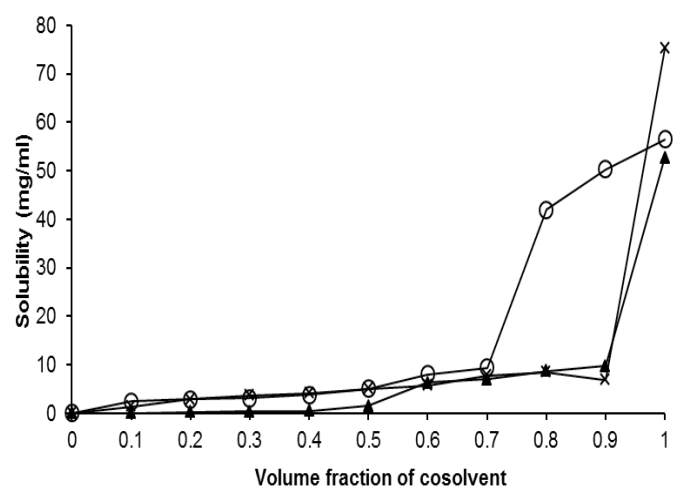

Figure 1: Solubility profile of modafinil in water-cosolvent mixtures; $E G(\mathbf{\Delta})$, PEG 400 (X), PEG 600 (O).

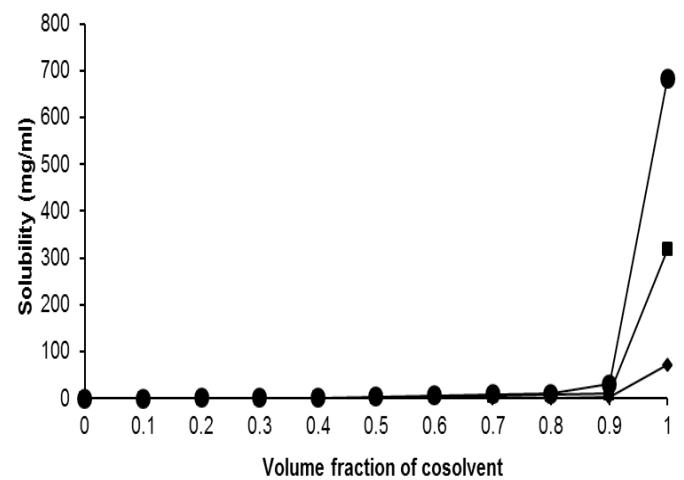

Figure 2: Solubility profile of modafinil in water-cosolvent mixtures; glycerin $(\bullet)$, PG $(\bullet)$, ethanol $(\bullet)$

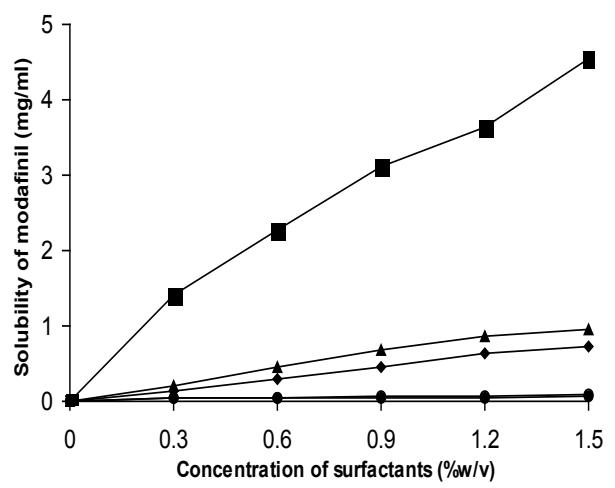

Figure 3: Solubility profile of modafinil in surfactants solutions; Tween 80 ( $\bullet$, Brij 35 (०), SLS ( $\mathbf{\Delta})$, Poloxamer $(\bullet)$, Cetrimide (๘).

and was concluded that the hydrocarbon core appears to have more solubilization capacity than polyoxyethylene mantle. ${ }^{24}$

The centre of solubilization in ionic surfactant is different from that of non-ionic surfactants. This is evidenced with the modafinil solubility in presence of sodium lauryl sulphate which being an ionic surfactant increased drug solubility linearly with its concentration. Further, it was noteworthy to mention that the higher solubility is noticed in presence of SLS in spite of its shorter hydrocarbon chain length than Brij 35. Outside palisade region of micelles is the centre for solubilization. ${ }^{25}$ This could be the mechanism for increased modafinil solubility in SLS solutions. 


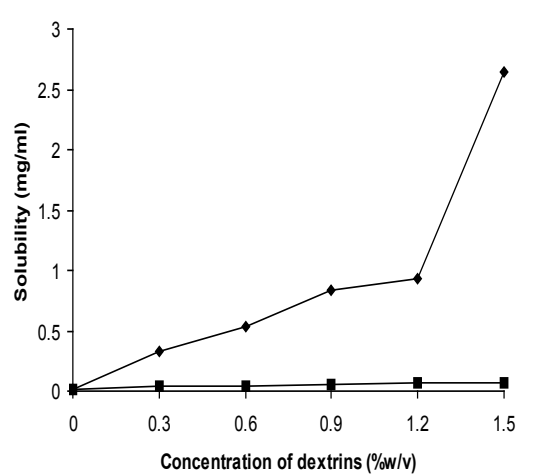

Figure 4: Solubility profile of modafinil in cyclodextrin solutions; $\beta$ - cyclo$\operatorname{dextrins}(\$)$, maltodextrins $(\mathbf{\square})$.

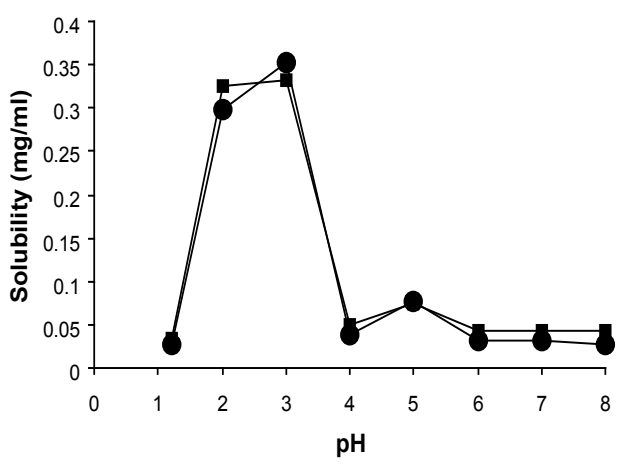

Figure 5: Solubility profile of modafinil in buffer solutions of $\mathrm{pH}$ ranging from 1.2 to 8 at $25^{\circ}(\bullet)$ and $370(\cdot)$.

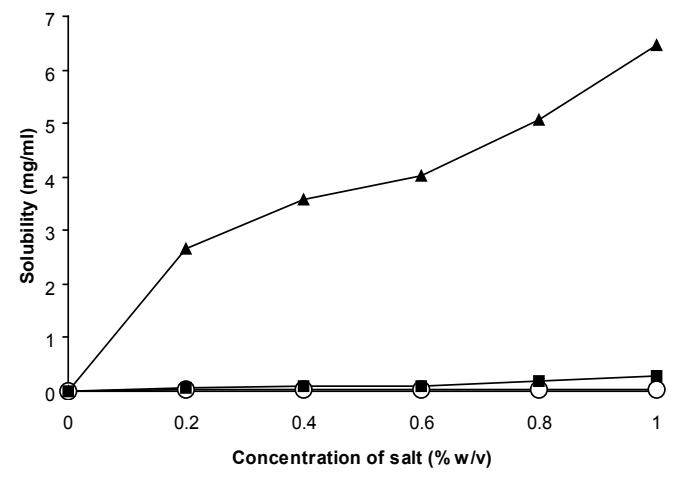

Figure 6: Solubility of modafinil in presence of different salts - $\mathrm{NaCl}(\bullet), \mathrm{NaBr}$ $(\mathbf{\square}), \mathrm{Na}_{2} \mathrm{SO}_{4}(0)$ and $\mathrm{NaSCN}(\mathbf{\Lambda})$.

Both the $\beta$-cyclodextrin (189-fold increments) and maltodextrin (5fold increment) showed noteworthy increase in modafinil aqueous solubility. Linearity obtained in the phase solubility diagrams (Figure 4) shows features of $A_{L}$-type of solubility curve. ${ }^{25}$ This is endorsed to formation of soluble complex. Stability constants calculated using the slope of the curve assuming the stoichiometry of theses complexes as 1:1 are shown in the Table 3. Hydrophobic molecules are complexed by cyclodextrins in a host-guest fashion. Either the whole drug or a part of the drug (guest) will be present in the central hydrophobic cavity (guest) of cyclodextrin. This process is supported by ejection of enthalpy-rich molecules of water from hydrophobic cavity that will not be sufficient for hydrogen bonding strength. The role of certain forces like van der Walls forces cannot be ruled out in the complex formation as evidenced by some scientists. ${ }^{26}$ Solubility enhancement of ebselen with several substituted betacyclodextrins is similar to the result obtained. ${ }^{27}$

It was suggested that hydrophobic character near the cavity played an important role in increasing the values of stability constant $(K) \cdot{ }^{27}$ Stability constant $\left(K_{c}\right)$ was calculated using the intercept and slope of solubility plot by the below typed equation;

$K_{c}=$ Slope $/ S_{o}$ (1-Slope)

The solubility of modafinil increases with increase in $\mathrm{pH}$ from 1.2 and maximum solubility is observed at 3 , thereafter the solubility decreases. It indicates that more amount of drug is in solubilized form in the stomach (acidic $\mathrm{pH}$ up to 3.0). In addition, the experimentally determined $\log P$ value of modafinil in $n$-octanol-water system is 5.3165 . In the stomach, the drug molecules easily penetrate through lipid membranes into the mucosal cell wall. The solubility of modafinil is slightly more at $\mathrm{pH} 5.0$ when compared to its neighboring $\mathrm{pH}$ values 4.0 and 6.0. This could be because of second ionization constant. Though $\mathrm{pKa}$ determination by spectrometric method was unsuccessful, by observing $\mathrm{pH}$ - solubility profile of modafinil, it can be expected that the dissociation constant could be around 3 .

All the salts used in the investigation increased the solubility of modafinil. The increase in the solubility of modafinil may be due to its binding capacity with the salt molecules where by it forms a complex with salts. The solubility of a non-electrolyte in an aqueous salt solution, at low concentration, is given by the below mentioned Setschenow's equation;

$\log S_{0} / S=k_{s} C$

Where $S$ is the solubility in pure water, $S$ is the solubility in salt solution of concentration $\mathrm{C}(\mathrm{moles} / \mathrm{l})$ and $k_{s}$ is the salting coefficient, which has a characteristic value for a given salt-non electrolyte pair. A positive value of $k_{s}$ corresponds to salting out $\left(S_{o}>S\right)$; if $k_{s}$ is negative, salting in is observed $\left(S_{\mathrm{o}}<S\right)$. Using Setchenow's equation the solubility powers are calculated and are reported in Table 3 . The values indicate that salting in effect has taken place in all the cases. The order of salting coefficients of various salts is $\mathrm{NaSCN}>\mathrm{NaBr}>\mathrm{NaCl}>\mathrm{Na}_{2} \mathrm{SO}_{4}$. Salting coefficients given in Table 3 indicates that sodium chloride and sodium sulphate have equal effects and therefore could not be differentiated in the Figure 6.

\section{CONCLUSION}

The present investigation evaluated and compared the effect of five solubilization techniques on the modafinil solubility enhancement. Among the solvents, ethanol improved maximum aqueous solubility. Cetrimide was most efficient surfactant in the group, as it improved the modafinil solubility by 324 times. Importantly, data reveal that centre of solubilization for modafinil is hydrophobic inner core of micelles when surfactants are used. In the class of cyclodextrins, $\beta$-cyclodextrin complexes increased the solubility by 189 folds with high stability constant. Buffer of $\mathrm{pH} 3$ gives maximum solubility. The NaSCN salt improved the water solubility by hydrotrophy to maximum extent among the salts used in the study. The entire investigation generated an important database of modafinil solubility using the techniques adopted in the pharmaceutical industry, which can be consulted while analyzing or formulating dosage forms of modafinil.

\section{ACKNOWLEDGEMENT}

The authors are grateful to Advance Research Wing, Rajiv Gandhi University of Health Sciences, Bangalore for funding this project. 


\section{CONFLICT OF INTEREST}

The authors declare no conflict of interest between the authors.

\section{REFERENCES}

1. Attwood D, Florence AT. Surfactant systems: Their chemistry, pharmacy and biology. London: Chapman and Hall. 1983.

2. Seedher N, Kanojia M. Co-solvent solubilization of some poorly-soluble antidiabetic drugs. Pharm Dev Technol. 2009;14(2):185-92.

3. Tayade P, Modi A. A comparative solubility enhancement profile of valdecoxib with different solubilization approaches. Indian J Pharm Sci. 2007;69(2):274-8.

4. Loftsson T, Brewster ME. Pharmaceutical applications of cyclodextrins: 1 Drug solubilization and stabilization. J Pharm Sci. 1996;85(10):1017-25.

5. Martin A. Physical pharmacy-physical chemical principles in the pharmaceutical sciences. $3^{\text {rd }}$ ed. Philadelphia: Lea and Febiger. 1993.

6. Loh GOK, Tan YTF, Peh KK. Enhancement of norfloxacin solubility via inclusion complexation with $\beta$-cyclodextrin and its derivative hydroxypropyl- $\beta$ cyclodextrin. Asian J Pharm Sci. 2016;11(4):536-46.

7. García A, Leonardi D, Salazar MO, Lamas MC. Modified $\beta$-cyclodextrin inclusion complex to improve the physicochemical properties of albendazole. Complete in vitro evaluation and characterization. PLoS One. 2014;9(2):e88234.

8. Yingqing R, Akash J, Yalkowsky SH. Solubilization and preformulation studies on PG-300995 (An Anti-HIV drug). J Pharm Sci. 2005;94(2):297-03.

9. Al-Maaich A, Flanagan DR. Salt effects on caffeine solubility, distribution and self-association. J Pharm Sci. 2002:91(4):1000-8.

10. Kalepu S, Nekkanti V. Insoluble drug delivery strategies: Review of recent advances and business prospects. Acta Pharm Sin B. 2015;5(5):442-53.

11. Higuchi T, Connors KA. Phase solubility techniques. Adv Anal Chem Instrum. 1965;4:117-22

12. Ainley W, Paul JW. Handbook of pharmaceutical excipients. $2^{\text {nd }}$ ed. London: The Pharmaceutical Press. 1994

13. Modi A, Tayade P. A comparative solubility enhancement profile of valdecoxib with different solubilization approaches. Ind J Pharm Sci. 2007;69(2):274-8.

14. Sheu M, Chen S, Chen L, Ho H. Influence of micelle solubilization by tocopheryl polyethylene glycol succinate (TPGS) on solubility enhancement and percutaneous penetration of estradiol. J Control Release. 2003;88(3):355-68.

15. Ikeda $\mathrm{K}$, Tomida $\mathrm{H}$, Yotsuyanagi T. Micellar interaction of tetracycline antibiotics. Chem Pharm Bull. 1977;25(5):1067-72.

16. Ochi $M$, Kimura $K$, Kanda A, Kawachi $T$, Matsuda $A$, Yuminoki $K$, et al. Physicochemical and pharmacokinetic characterization of amorphous solid dispersion of meloxicam with enhanced dissolution property and storage stability. AAPS Pharm Sci Tech. 2016;17(4):932-9.

17. Satheshbabu PR, Subrahmanyam CVS, Thimmasetty J, Manavalan R, Valliappan K. Solubility of meloxicam in mixed solvent systems. Ethiop Pharm J. 2007;25(1):23-8.

18. Philip HH, William MM. Handbook of physical properties of organic chemicals. Boca Raton: Lewis Publishers. 1997.

19. Ikeda H, Chiba K, Kanou A, Hirayama N. Prediction of solubility of drugs by conductor like screening model for real solvents. Chem Pharm Bull. 2005;53(2):253-5

20. Nayak AK, Panigrahi PP. Solubility Enhancement of etoricoxib by cosolvency approach. ISRN Physical Chemistry. 2012. [Cited 2017 July 10]; Article ID 820653. Available from: https://doi.org/10.5402/2012/820653.

21. Ran $Y$, Zhao L, Xu Q, Yalkowsky SH. Solubilization of cyclosporin A. AAPS Pharm Sci Tech. 2001;2(1):23-6.

22. Jain AK. Solubilization of indomethacin using hydrotropes for aqueous injection. Eur J Pharm Biopharm. 2007;68(3):701-14

23. Kadam PS, Pande VV, Vibhute SK, Giri MA. Exploration of mixed hydrotropy strategy in formulation and development of etodolac injection. J Nanomed Res. 2016;3(4):63.

24. Moro ME, Velazquez MM, Cachaza JM, Rodriguez LJ. Solubility of diazepam and prazepam in aqueous nonionic surfactants. J Pharm Pharmacol. 1986; 38(4):294-6.

25. Alkhamis KA, Hussien A, Nawzat DAZ. Effect of surfactant on dissolution of spherical particles in micellar systems. Eur J Pharm Biopharm. 2007;65(2):18897.

26. Connors KA. The stability of cyclodextrin complexes in solution. Chem Rev. 1997:97(5):1325-58

27. Nagase $Y$, Suzuki N, Yamauchi $H$, Kim S, Wada $K$, Arima $H$, et al. Inclusion complexation of a selenoorganic antioxidant abselen with cyclodextrin in aqueous solution. J Incl Phenom Macro Chem. 2002;44(1-4):107-10.

Article History: Submission Date : 01-01-2020; Revised Date : 20-02-2020; Acceptance Date : 10-03-2020.

Cite this article: Thimmasetty J, Ghosh T, Nayak SN, Kamath S, Seetharaman S, Abbas KM. Enhanced Solubility of Modafinil via Solubilization Techniques. J Young Pharm. 2020;12(2):129-34. 